\title{
Experimental observations of longitudinal space-charge waves in intense electron beams
}

\author{
K. Tian, Y. Zou, Y. Cui, I. Haber, R. A. Kishek, M. Reiser, and P. G. O’Shea \\ Institute for Research in Electronics and Applied Physics, University of Maryland, College Park, Maryland, 20742, USA
}

(Received 6 September 2005; published 25 January 2006)

\begin{abstract}
Studies of the dynamics of longitudinal space-charge waves in space-charge dominated beams propagating through a transport channel with a long solenoid are performed at the University of Maryland. In this paper, we report some experimental results on the energy modulations converted from density modulations. By changing the working conditions of the electron gun, pure initial density modulations are generated. Energy perturbation waveforms are measured with a high-resolution energy analyzer. The experimental results are compared with both the linear theory and the simulation results. Good agreements are achieved for the relationship between the energy and current perturbation strengths.
\end{abstract}

DOI: 10.1103/PhysRevSTAB.9.014201

PACS numbers: 29.27.Bd, 29.27.Fh, 29.30.Dn

\section{INTRODUCTION}

Charged particle beams have been used in many diverse areas of scientific research and industrial applications [1], such as electron microscopes, cathode ray tubes (CRT) and particle accelerators. The physics of low intensity beams has been well studied since the 1920s. However, the recent interest in heavy ion fusion (HIF) [2], the developments of the spallation neutron source [3], free electron lasers (FEL) [4], and injector linacs for future high-energy physics colliders with high luminosity require more detailed knowledge and understanding of the physics of the high intensity beams in which space-charge forces play a much more important role than in conventional accelerators, such as the existing high-energy collider rings. In the spacecharge dominated beams, the nonlinear space-charge forces will introduce many collective effects, which may limit the maximum current or beam quality. Some of these collective behaviors are not well understood. One of the effects is the physics of longitudinal space-charge waves, which can be generated by density perturbations or energy perturbations caused by many factors, such as the discontinuity of the beam transport modules, fluctuations in the bunch, or the mismatch of the focusing channels. These perturbations tend to excite longitudinal instabilities in the beam that are caused by interacting with the external transport and acceleration environments. Energy dispersion caused by the energy modulations of space-charge waves will also introduce troubles in some high intensity machines. Theoretical investigations on the evolutions of space-charge waves have been carried out since the 1980s [5-7]. The most important analysis of the space-charge waves is the linear theory based on a one-dimensional cold fluid theory. In this theory, with small initial perturbations, momentum and continuity equations are solved. The solutions show that the modulations travel along the beam in the form of waves called space-charge waves and are the superposition of two eigenfunctions. One of them has a phase velocity greater than the main beam velocity, so it is called the fast space-charge wave. The other has a phase velocity smaller than the main beam velocity, so it is called the slow space-charge wave. In order to get the analytical solution for the space-charge waves, the initial conditions and boundary conditions for the linearized momentum and continuity equations are assumed as follows: there is no perturbation anywhere along the longitudinal direction when $t$ is less than zero; at $z=0$ and $t=0$, a localized velocity perturbation and current perturbation are introduced. The initial perturbations are in the form

$$
\begin{aligned}
& v_{1}(0, t)=\delta v_{0} h(t) \\
& I_{1}(0, t)=\eta I_{0} h(t) .
\end{aligned}
$$

$I$ and $v$ are the current and velocity of the beam respectively; the subscript 0 and 1 represent the unperturbed and the perturbed values, respectively; $\delta$ is a small, positive quantity to specify the strength of the velocity perturbation; $\eta$ is a small quantity to specify the strength of the initial current perturbation and can be negative if the velocity increase causes a current decrease. $h(t)$ is any smooth function with amplitude of unity, which represents the shape of the perturbation and is supposed to vanish when $t$ is equal to or smaller than zero. Thus, with the omission of higher-order terms, the line charge density perturbation $\Lambda$ can be expressed as

$$
\Lambda_{1}(0, t)=(\eta-\delta) \Lambda_{0} h(t) .
$$

The solutions of the linearized continuity and momentum transfer equations are

$$
\begin{gathered}
\Lambda_{1}(z, t)=-0.5 \Lambda_{0}[\delta V-(\eta-\delta)] h\left(t-t_{s}\right) \\
+0.5 \Lambda_{0}[\delta V+(\eta-\delta)] h\left(t-t_{f}\right), \\
v_{1}(z, t)=0.5 v_{0}[\delta-(\eta-\delta) / V] h\left(t-t_{s}\right) \\
+0.5 v_{0}[\delta+(\eta-\delta) / V] h\left(t-t_{f}\right), \\
I_{1}(z, t)=-0.5 I_{0}[\delta V-\eta+(\eta-\delta) / V] h\left(t-t_{s}\right) \\
+0.5 I_{0}[\delta V+\eta+(\eta-\delta) / V] h\left(t-t_{f}\right) .
\end{gathered}
$$


Here, $V=v_{0} / c_{s} ; t_{s}=z /\left(v_{0}-c_{s}\right) ; t_{f}=z /\left(v_{0}+c_{s}\right)$; $c_{s}$ is called "sound speed," which is the velocity of hspace-charge waves in the beam frame, in analogy to the propagation of sound waves in a gas. All these expressions have two terms: the first term is the slow wave; the second term is the fast wave. Both the fast wave and the slow wave keep the shape of the initial perturbation, while the amplitude and polarity are decided by the initial conditions.

\section{LONG SOLENOID EXPERIMENT SETUP}

A $2.5 \mathrm{~m}$ long beam transport line was set up at the University of Maryland to study the longitudinal spacecharge perturbation and energy spread evolution in a long uniform focusing channel. The schematic of the entire system is shown in Fig. 1. A thermionic gridded electron gun [8] is used as the source of the electron beams. A highresolution energy analyzer [9-11] is installed in the system. The long solenoid M4, which is $1.4 \mathrm{~m}$ long, serves as a uniform focusing channel to transport the beam into the energy analyzer. In order to match the beam into the long solenoid, three short solenoids, M1, M2 and M3, are placed in the system. Another short solenoid M5 is placed between the exit of the long solenoid and the energy analyzer in order to control the amount of current injected into the energy analyzer. The axial magnetic field profiles along the axis of these solenoids were measured with a Hall probe. The fields off axis can be obtained using the formulas in Ref. [1] derived from Taylor expansion. One Bergoz fast current transformer is located between solenoids M1 and M2. A very high vacuum at low $10^{-8}$ to high $10^{-9}$ Torr is maintained by four ion pumps. The first ion pump is located at the electron gun with a capacity of $81 / \mathrm{s}$. The other three ion pumps have capacities of $40 \mathrm{l} / \mathrm{s}$. One is located between the second and third solenoids and two are located at the diagnostic chamber. The beam pipeline is connected with an automated measurement system including a high-voltage power supply, a Tektronix oscilloscope (TEK DSA 601A) and a computer. A MATLAB code was developed to guide the setting of the currents of the solenoids for matching the electron beams into the long solenoid.

The basic idea of this experiment is to observe the energy perturbation, which is excited by a pure current perturbation. In order to produce a pure current modulation at the electron gun, which has a triode structure, a cable is connected to the middle of the pulse generation transmission line through a " $T$ " connector. As a result, a perturbation is generated at the center of the voltage pulse, which can introduce a perturbation to the beam current. The main beam energies in all the experiment are $5 \mathrm{keV}$, while the

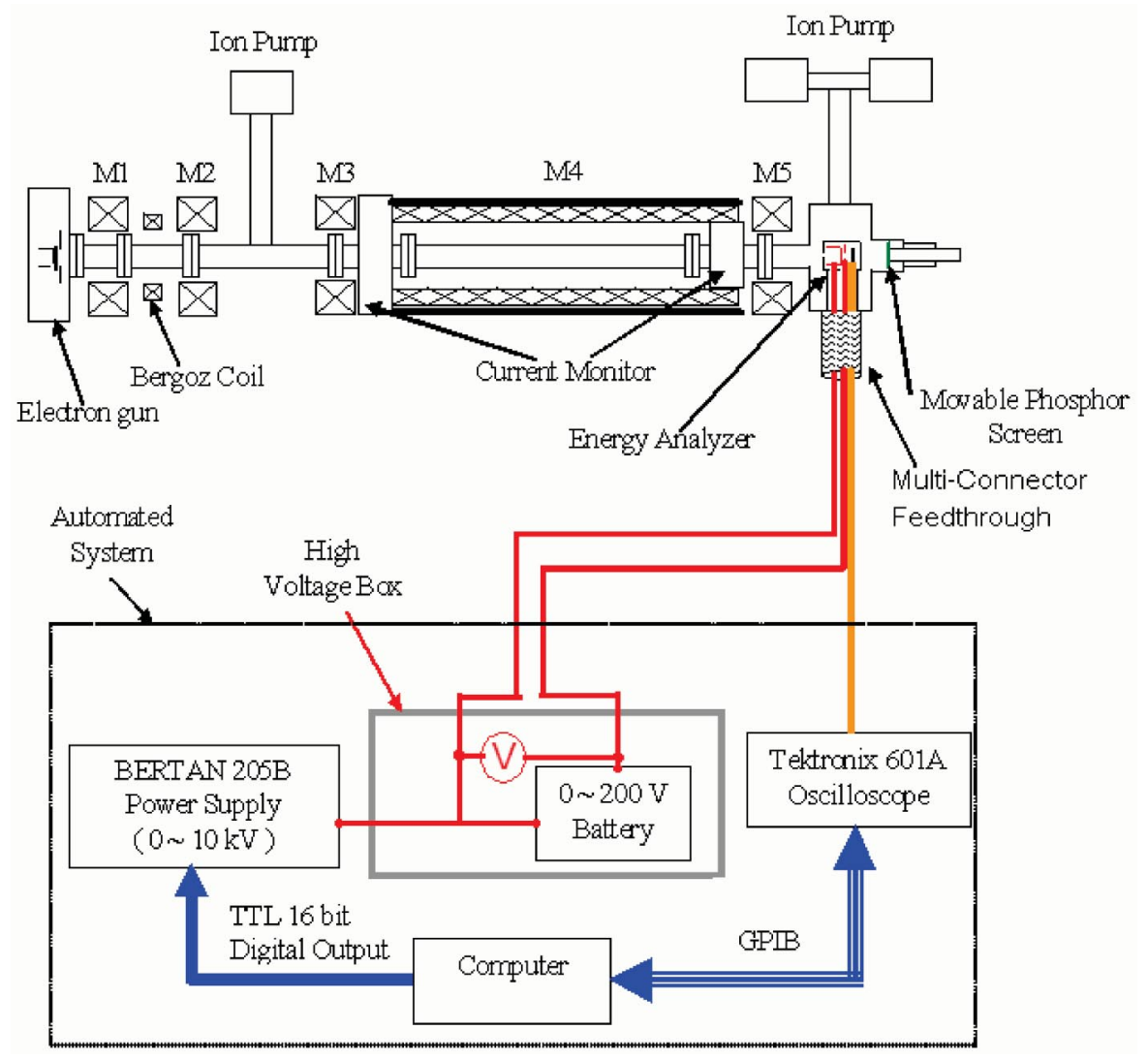

FIG. 1. (Color) Schematic of the long solenoid experiment system setup. 
TABLE I. Data for current perturbation generation at the gun.

\begin{tabular}{lcccc}
\hline \hline Group number & Bias voltage/V & Main current/mA & Current perturbation/mA & Perturbation strength/ $\eta$ \\
\hline 1 & 2 & 69.6 & 3.97 & 0.057 \\
2 & 14 & 77.4 & 10.85 & 0.137 \\
3 & 30 & 67.2 & 16.00 & 0.226 \\
4 & 32 & 45.6 & 13.20 & 0.29 \\
\hline \hline
\end{tabular}

relative strengths of the voltage perturbations are in the order of $10^{-3}$, which is very small compared to the initial perturbation strengths of currents; hence in most cases, the initial energy modulations created by the voltage pulses can be neglected. Using a laser to impinge the photocathode can generate pure initial current perturbations more accurately $[12,13]$. We may use this method in future experiments. According to the theoretical predictions, both current and velocity perturbations will exist in the form of a fast wave and a slow wave at the downstream of the transport line. The energy analyzer can measure the energy perturbation waveform after the long solenoid. The current perturbations can be measured using the Bergoz current monitor. In order to generate different strengths of current perturbations, we change the bias voltage of the electron gun. One should notice that the main beam currents are also changed with this method. Four groups of electron beams with different main beam currents and different strengths of current perturbations are achieved, as shown in Table I. The current profiles in Fig. 2 are acquired from the Bergoz current monitor between matching solenoids M1 and M2.

\section{EXPERIMENTAL RESULTS}

The energy spectrum at different positions in the beam can be measured with the high-resolution energy analyzer. Therefore, the mean energy along the beam can be calculated easily by integrating the energy spectrum. In Fig. 3, we plot the mean energy along the beam at a distance of $2.3 \mathrm{~m}$ after the gun for different groups. As the theory predicts, with the initial condition of pure density modulation, energy modulation can be achieved, which split into two peaks at the beam center: one is positive and near to the beam head, corresponding to the fast wave, the other is a negative peak and near to the beam end, corresponding to the slow wave. According to the one-dimensional cold fluid theory, the peaks of the fast wave and the slow wave should have the same amplitude. However, here we see some inconsistency with the theoretical predictions.
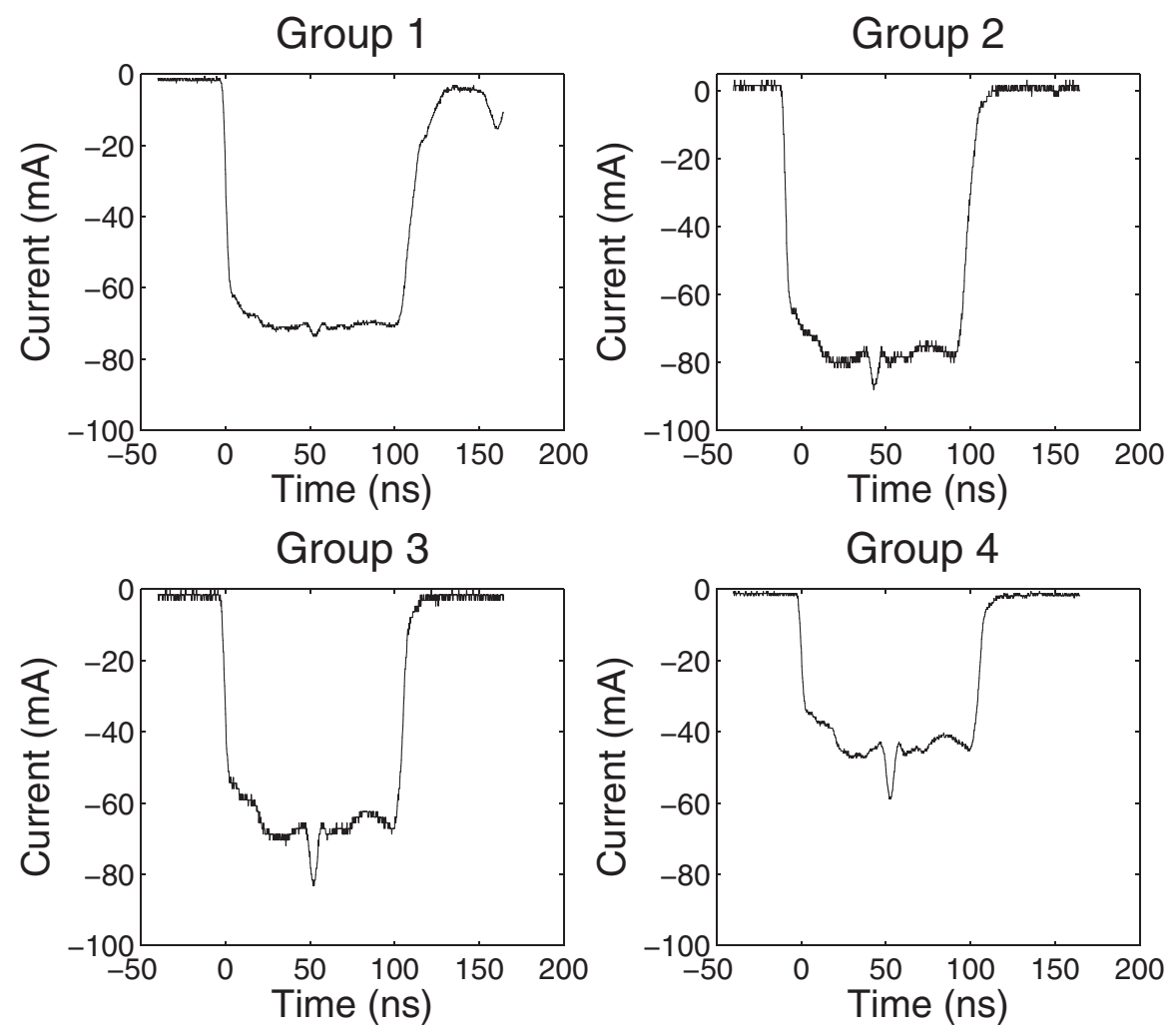

FIG. 2. Four groups of initial currents with perturbation. 

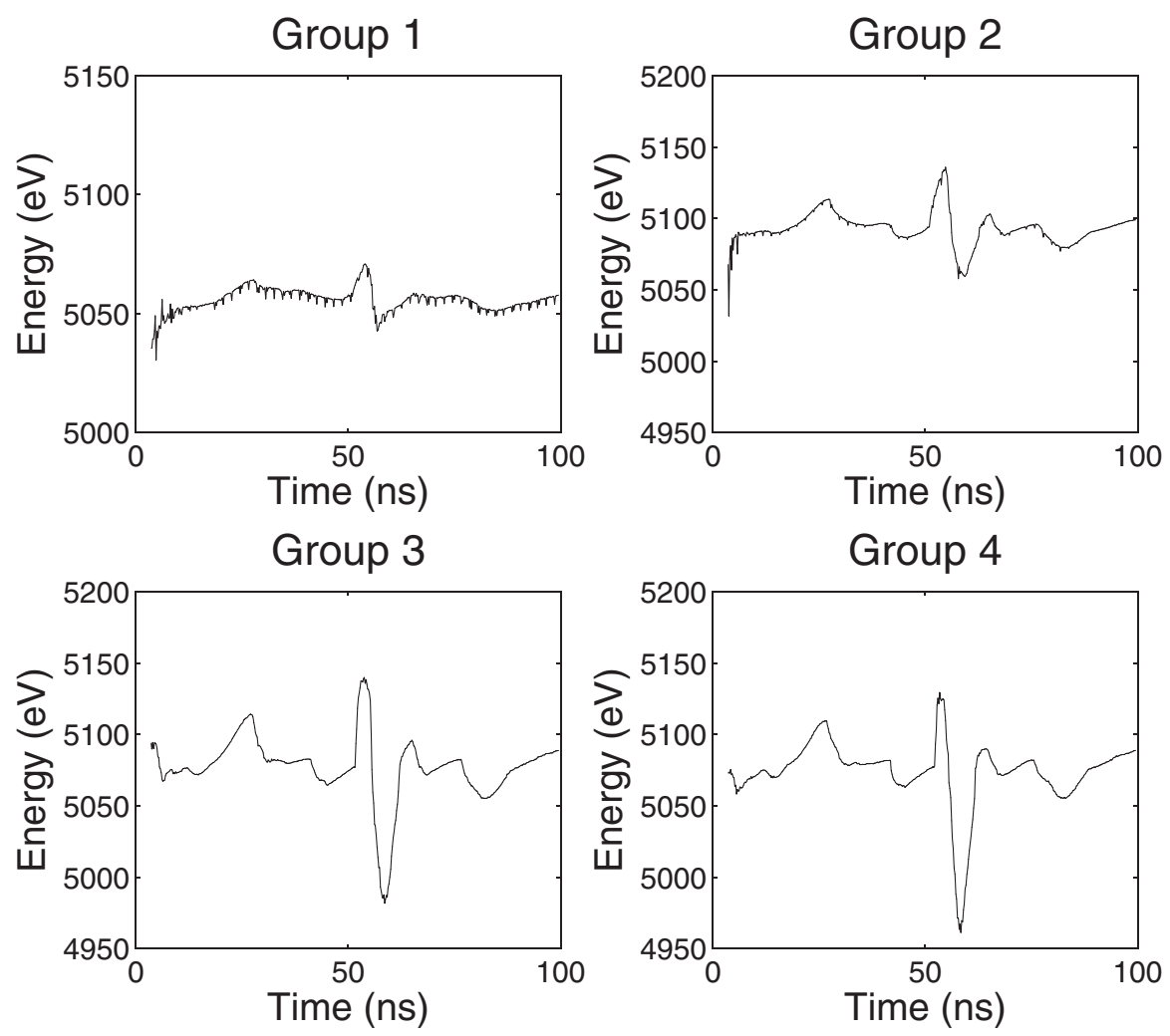

FIG. 3. Mean energy waveforms derived from energy analyzer signals for different groups.

Especially for the result of group 4, the amplitude of the slow wave is much larger than that of the fast wave. The reason for this is not clear up to now. However, it may be related to the following aspects: First, for groups 3 and 4, the current perturbations are more than $20 \%$ of the main beam current, so nonlinear effects should not be neglected, while the one-dimension theory has the assumption of linearity, which implies that the strength of the perturbations must be small in comparison with the beam current, say $<10 \%$. Second, during the experiments, the beams are not stable enough, which also causes errors of the measurements. Another observation is that some other peaks are obtained for these four groups of results. Compared with the initial currents in Fig. 2, it is apparent that they are due to the bumps near the beam head and beam end. Here we only focus on the peaks for the slow wave and fast wave at the beam center.

\section{SIMULATION RESULTS AND COMPARISONS}

We use an automated data acquisition system so that the computer can acquire the current profiles measured by the Bergoz coil. This makes it fast and very convenient to import the experimental values of the beam currents, generated by the electron gun, into WARP [14], a particlein-cell code. WARP is then used to simulate the beam propagation. In the following paragraph, we will describe this procedure in more detail.
First, the current profiles of the Bergoz coil are imported into the WARP code to serve as the initial conditions for the simulation. Here, the data of group 3 are taken as an example to illustrate this process. A considerable amount of digital noise is observed in the current profiles. In order to reduce the noise, the data are smoothed before the simulation in WARP starts. The curve in Fig. 4 is the current

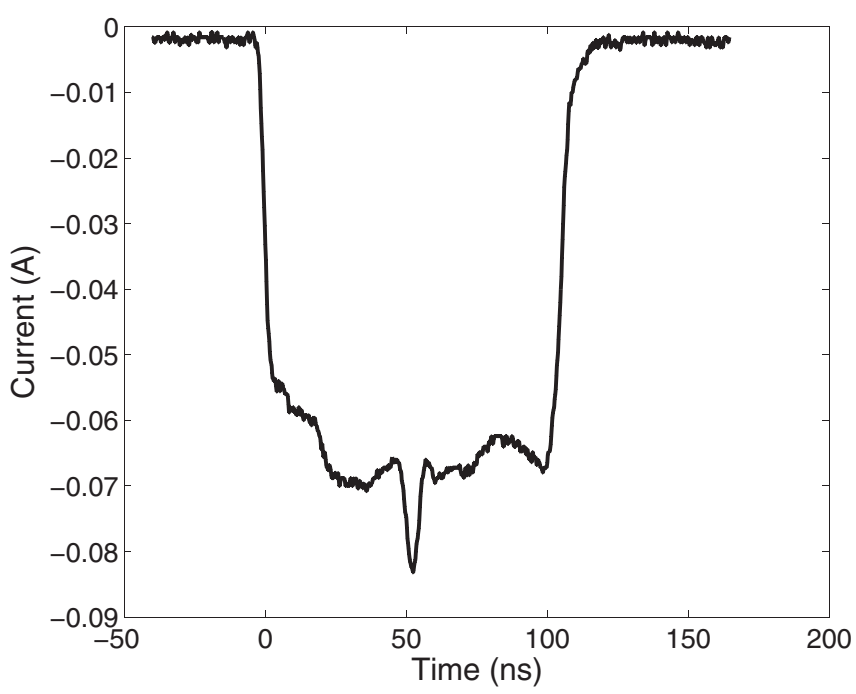

FIG. 4. Current profile imported into WARP in group 3. 
TABLE II. Beam parameter settings in WARP.

\begin{tabular}{lccc}
\hline \hline Group number & $I_{0} / \mathrm{mA}$ & $E_{0} / \mathrm{eV}$ & $R / \mathrm{mm}$ \\
\hline 1 & 69.6 & 5055 & 5.3 \\
2 & 79.2 & 5092 & 5.6 \\
3 & 67.2 & 5074 & 5.2 \\
4 & 45.6 & 5077 & 4.4 \\
\hline \hline
\end{tabular}

profile after the smoothing procedure. The energy perturbation is set to zero initially in WARP.

Then, the parameters such as beam radius $R$, main beam current $I$, and main beam kinetic energy $E$ are set up in the WARP code. The beam radius is calculated by a matching code in MATLAB using the magnetic field strength of the solenoid channel from the experiment [15]. As shown in Fig. 1, the distance of $2.3 \mathrm{~m}$ is larger than the length of the long solenoid. In the WARP simulations, a "smooth uniform field" corresponding to that of the long solenoid was used to represent the magnetic field variation along this channel. The comparison between measurements and simulations show that this approximation has only a negligible effect on our results. The initial transverse distribution of the electron beams in WARP is a semi-Gaussian distribution, which is uniform in space and Gaussian in velocity space, with a uniform temperature. Different settings for each group are shown in Table II. Other numerical settings for simulation are: the length of the beam pulse is $100 \mathrm{~ns}$; the particle number is 50000; the number of cells in the $r$ direction is 64; the number of cells in the $z$ direction is 256 .

After the initial settings, the simulations are carried out in the $r-z$ geometry for a channel length of $2.3 \mathrm{~m}$, which is the distance between the Bergoz coil and the retarding field energy analyzer in the experiment. In order to compare the results with the one-dimensional linear theory, the analytical solutions for each group are solved based on the formulas (4) and (5). All the results from WARP, the onedimensional theory and the experiment are shown in Fig. 5. The WARP results are represented by the red curve; the experimental results are represented by the black curve; the one-dimensional cold fluid theory predictions are represented by the green curve.

From these curves, the similar shapes of the energy perturbations for WARP simulation, experiment results and the one-dimensional cold fluid theory can be observed in each group. Except for the fast wave and slow wave generated near the beam center, some other perturbation peaks with positive and negative polarity can be observed near the beam head and beam tail.

One can also observe from Fig. 5 that the amplitudes of the energy perturbations are not equal for different calculation methods in each group. The space-charge waves were transported for only $2.3 \mathrm{~m}$ before they are measured, so the fast wave and slow wave due to the current pertur-
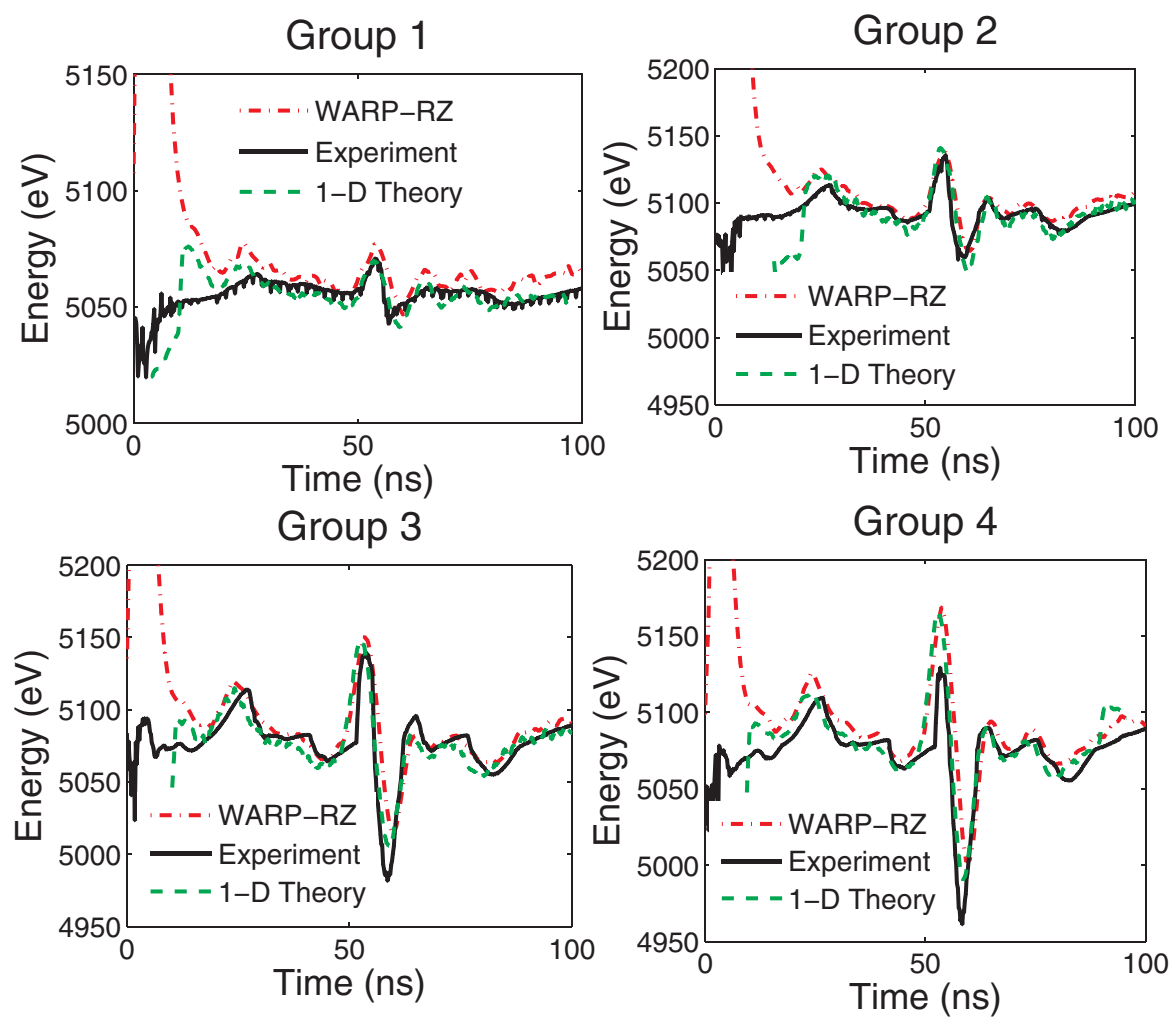

FIG. 5. (Color) Comparisons of the profiles of the mean energy for the experiment results, WARP simulation results and onedimensional cold fluid theory. 
bation at the beam center may not separate completely. This indicates that these peaks can be smaller than those derived after the pair of space-charge wave separate completely. Another problem is the fact that the main beam current is not as flat as the ideal beam pulse and has some bumps, which introduce additional current perturbations along the beam other than the perturbation generated at the center of the beam. We cannot observe the complete separation of the slow wave and fast wave even after the beam in the simulation is transported for a longer distance. Therefore, the perturbations we observed are always the superposition of slow waves and fast waves.

One major concern was whether our results depend on the beam radius and hence on the extent of transverse mismatch. To answer this question, we performed WARP simulation studies for group 3. The mean energy and the current remained the same as those in Table II, but the radius was changed to $150 \%, 120 \%, 80 \%$, and $50 \%$ of the matched beam radius of $5.2 \mathrm{~mm}$. The results of these WARP simulation runs are plotted in Fig. 6, which clearly shows that they are insensitive to transverse beam mismatch.

In order to quantitatively compare the experimental results with simulation and theory, it is useful to compare the peak-to-peak values of the energy waveforms, which are defined as the difference between the peak values of the fast wave and the slow wave. The values for the energy perturbation peak-to-peak strengths $\Delta E / E_{0}$ measured at $z=2.3 \mathrm{~m}$ using different methods and initial current perturbation strengths $\eta$, are shown in Table III.

Figure 7 shows the relationship between $\Delta E / E_{0}$ and $\eta$ based on the data in Table III. The black dots represent experiment data, the green squares represent the WARP

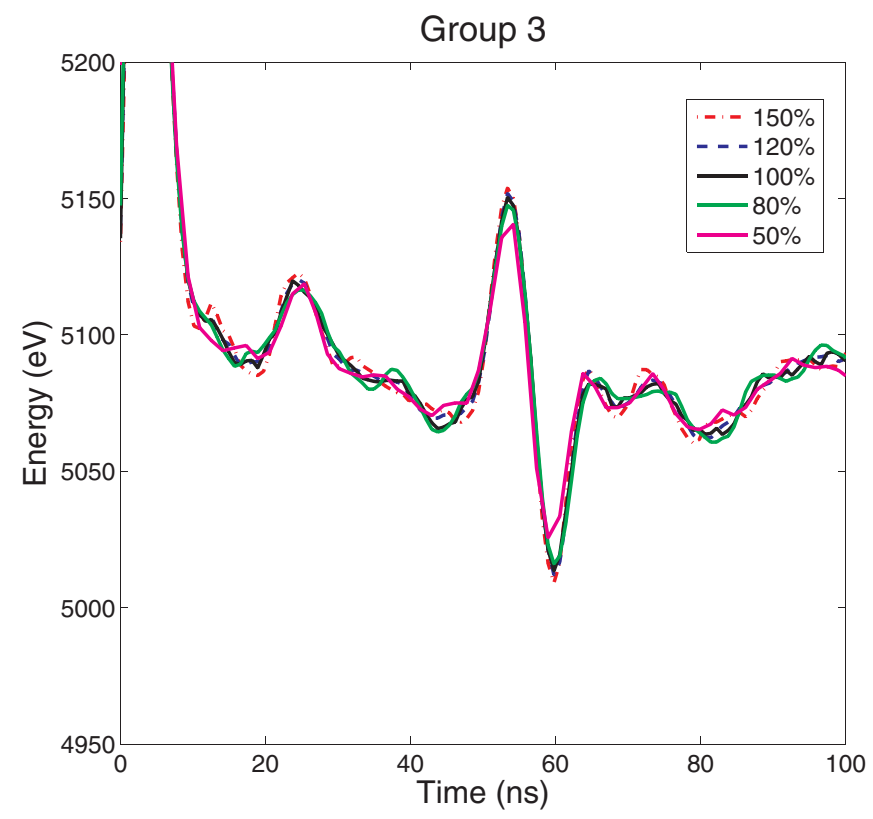

FIG. 6. (Color) The results of mean energy vs time for different beam radius in WARP.
TABLE III. Energy perturbation peak-to-peak strengths and $\eta$.

\begin{tabular}{lccc}
\hline \hline & & $\Delta E / E_{0}$ \\
\hline$\eta$ & Experiment & WARP & 1D theory \\
\hline 0.057 & 0.0056 & 0.006 & 0.0056 \\
0.137 & 0.0152 & 0.0144 & 0.0179 \\
0.226 & 0.0312 & 0.027 & 0.0283 \\
0.29 & 0.0331 & 0.0328 & 0.0345 \\
\hline \hline
\end{tabular}

simulation results, and the red stars represent analytical solution from the one-dimensional cold fluid theory. The experimental results show very good agreement with both WARP simulation and analytical results based on the onedimensional cold fluid theory. All these results show that the energy perturbation peak-to-peak strength $\Delta E / E_{0}$ grows linearly with the current perturbation strength $\eta$, and the slope is about 0.1. Using the least square method, the slope can be calculated: for the experiment data, which yields 0.127; for the WARP simulation results it is 0.119 , and for the one-dimensional theoretical prediction, it is 0.124 . Therefore, the conclusion can be drawn that good predictions of the energy perturbation strength evolution can be derived from the WARP simulation and the one-dimensional cold fluid theory. For groups 3 and 4, the current perturbation strength $\eta$ is equal to 0.0226 and 0.29 , respectively, which are not as small as is assumed in the linear theory. However, the velocity perturbation in our case is very small. As a result, the product of the current perturbation and velocity perturbation can still be neglected and the formula (3) is still applicable. Thus, it is reasonable to say that the linear theory agrees with our experimental and simulation results. However, the asymmetry pattern of the slow wave and fast wave from experimental observation in

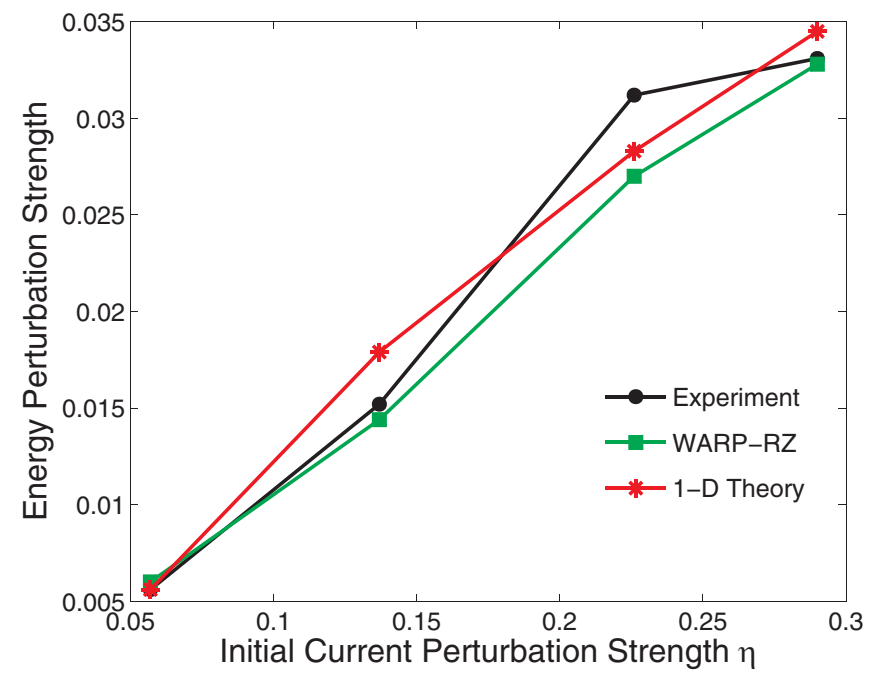

FIG. 7. (Color) The relationship between the strength of the energy perturbation and the initial strength of the current perturbation. 
group 4 of Fig. 5 shows inconsistency with the linear theory and the WARP simulation. One possible reason for this problem may be due to the discrepancy between the real beam current profiles and the initial current profiles used in the WARP simulation from the Bergoz coil measurement, about $20 \mathrm{~cm}$ from the gun. We assumed a zero initial energy perturbation in WARP. However, these assumptions may not be correct at the location of the Bergoz coil where the initial current profiles are different from the real current profiles at the gun. We have modified the experiment system, and a new energy analyzer will be installed between solenoid M2 and M3. The two energy analyzers in the system should help us shed some light on this issue. Another possible reason may be due to our assumption of the initial condition of pure density modulation, in which the initial energy is neglected. One method, which we may pursue in the future, is to generate pure initial density modulation by using a laser to impinge on the photocathode, as discussed in Sec. II.

\section{SUMMARY}

Experiments on the evolution of an initial pure current modulation are performed in the long solenoid experiment system at the University of Maryland. This system includes a $1.4 \mathrm{~m}$ long solenoid, after which a new generation of retarding field energy analyzer is installed. Four groups of localized pure current perturbations with different strengths are generated by modulating the grid voltage at the electron gun and then transported through the matching solenoid to the uniform focusing channel. The kinetic energy of the beam is measured after the long solenoid. As the theory predicts, the space-charge waves of kinetic energy appear in pairs, one is a fast wave with a positive polarity, and the other is a slow wave with a negative polarity. In order to compare the experimental results with WARP simulations, the initial current waveforms are imported into the WARP code and the beam parameters are used consistently with the experiment. The energy perturbation peak-to-peak strength is defined in order to compare the experimental results of the amplitude of the energy waves with the WARP result and the analytical solution from the linear theory. The comparison shows that the peak-to-peak strength of the energy perturbations agrees very well with the experiment, WARP simulation and onedimensional cold fluid theory. However, some unexplained phenomena are observed in the experimental results, which require further work.

\section{ACKNOWLEDGMENTS}

The authors are grateful to Dr. Mark T. Walter and Mr. B. Quinn for their excellent mechanical and electrical support. We also want to thank Alex Friedman, Dave P. Grote, and Jean-Luc Vay for providing us with the WARP code. This work is supported by U.S. Department of Energy Grants No. DE-FG02-94ER40855 and No. DE-FG0292ER54178.

[1] M. Reiser, Theory and Design of charged Particle Beams (Wiley, New York, 1994).

[2] C. M. Celata, in Proceedings of XXth International Linac Conference, Monterey, CA, 2000, edited by A. W. Chao (SLAC Report No. SLAC-R-561), p. 668.

[3] J. Wei et al., in Proceedings of IEEE Particle Accelerator Conference, Portland, OR, 2003 (IEEE, Piscataway, NJ, 2003), p. 571.

[4] T.C. Marshall, Free Electron Laser (Macmillan Publishing Co., New York, 1985).

[5] J. G. Wang et al., Phys. Rev. Lett. 71, 1836 (1993).

[6] J. G. Wang et al., Phys. Rev. Lett. 74, 3153 (1995).

[7] Y. Zou, Ph.D. thesis, University of Maryland, 2000.

[8] J. G. Wang et al., IEEE Trans. Electron Devices 37, 2622 (1990).

[9] Y. Cui et al., Rev. Sci. Instrum. 75, 2736 (2004).

[10] Y. Zou, Phys. Rev. ST Accel. Beams 5, 072801 (2002).

[11] Y. Zou et al., Phys. Rev. Lett. 94, 134801 (2005).

[12] Y. Huo, Master's thesis, University of Maryland, 2004.

[13] J.G. Neumann et al., Rev. Sci. Instrum. 76, 033303 (2005).

[14] D. P. Grote, Fusion Eng. Des. 32-33, 193 (1996).

[15] Y. Cui, Ph.D. thesis, University of Maryland, 2004 